\title{
Tanshinone IIA attenuates heart failure via inhibiting oxidative stress in myocardial infarction rats
}

\author{
RUIJUAN CHEN $^{1 *}$, WENLI CHEN $^{2 *}$, XIAOLING HUANG $^{1}$ and QINGLIN RUI ${ }^{1}$ \\ ${ }^{1}$ Emergency Department, Affiliated Hospital of Nanjing University of Chinese Medicine, \\ Jiangsu Province Hospital of Chinese Medicine, Nanjing, Jiangsu 210029; \\ ${ }^{2}$ Department of Rehabilitation Medicine, Zhongda Hospital Southeast University, \\ Nanjing, Jiangsu 210009, P.R. China
}

Received June 24, 2020; Accepted December 22, 2020

DOI: $10.3892 / \mathrm{mmr} .2021 .12043$

\begin{abstract}
The purpose of the present study was to evaluate whether tanshinone IIA (TIIA) could treat cardiac dysfunction and fibrosis in heart failure (HF) by inhibiting oxidative stress. An HF model was induced by ligation of the left anterior descending artery to cause ischemia myocardial infarction (MI) in Sprague-Dawley rats. Cardiac fibrosis was evaluated using Masson's staining, and the levels of collagen I, collagen III, TGF- $\beta, \alpha$-smooth muscle actin ( $\alpha$-SMA), matrix metalloproteinase (MMP) 2 and MMP9 were determined using PCR or western blotting. TIIA treatment reversed the decreases of left ventricular (LV) ejection fraction, fractional shortening (FS), LV systolic pressure and the maximum of the first differentiation of $\mathrm{LV}$ pressure $\left(\mathrm{LV} \pm \mathrm{dp} / \mathrm{dt}_{\max }\right)$, the increases of $\mathrm{LV}$ volume in systole, LV volume in diastole, LV end-systolic diameter and LV end-diastolic diameter in MI rats. TIIA administration also reversed the increases of expression levels of collagen I, collagen III, TGF- $\beta, \alpha-$ SMA, MMP2 and MMP9 in the heart of MI rats and in angiotensin (Ang) II-treated cardiac fibroblasts (CFs). TIIA reversed the decreases of superoxide dismutase activity and malondialdehyde and the increases of superoxide anions and NADPH oxidase (Nox) activity in both MI rats and Ang II-treated CFs. Nox4 overexpression inhibited the effects of TIIA of improving cardiac dysfunction and fibrosis in MI rats and Ang II-treated CFs. These results demonstrated that TIIA improved cardiac dysfunction and fibrosis via inhibiting oxidative stress in HF rats. Nox4 could
\end{abstract}

Correspondence to: Dr Qinglin Rui, Emergency Department, Affiliated Hospital of Nanjing University of Chinese Medicine, Jiangsu Province Hospital of Chinese Medicine, 155 Hanzhong Road, Nanjing, Jiangsu 210029, P.R. China

E-mail: rql1964jsszyy@163.com

*Contributed equally

Key words: tanshinone IIA, heart failure, myocardial infarction, oxidative stress, fibrosis regulate the inhibitory effects of TIIA on $\mathrm{HF}$ and cardiac fibrosis.

\section{Introduction}

Heart failure (HF) remains a globally epidemic cardiac disease $(1,2)$. HF is preceded by left ventricular (LV) remodeling, such as enlarged myocardial size or increased LV mass due to pressure-overload (3). Myocardial infarction (MI), the coronary artery disease, can increase the risk of HF and is even life-threatening (4).

LV remodeling is characterized by increased cardiac interstitial fibrosis resulting from the accumulation of collagen type I, collagen type III, matrix metalloproteinase (MMP) 2, MMP9, TGF- $\beta$ and $\alpha$-smooth muscle actin ( $\alpha$-SMA) (5-7). Cardiac fibrosis is common in many cardiovascular diseases, such as myocardial infarction (8), hypertension (9) and cardiomyopathy (10) and is critical to the evolution of structural LV remodeling and the development of HF. Currently, the mechanisms of cardiac fibrosis in HF remains to be elucidated.

Tanshinone IIA (TIIA) is a natural compound extracted from the roots of Salvia miltiorrhiza Bunge and has been used in traditional Chinese medicine to protect against organ injuries (11). Studies have demonstrated that TIIA exhibits antioxidative, anti-cancer and anti-inflammatory effects (12-14). TIIA is also been used to treat cardiovascular diseases (15) because it can increase coronary blood flow and ameliorates myocardial metabolic disorder induced by hypoxia (16). Furthermore, TIIA treatment can reduce the infarction and increase myocardial regeneration and contractility (16). However, the effects of TIIA on HF remain to be elucidated.

Oxidative stress serves important roles in pathological cardiac remodeling and cardiac failure $(17,18)$. Coronary ligation can result in dilatation of left ventricle with compensatory hypertrophy of the remaining LV myocardium and the right ventricle (19). This remodeling is associated in LV non-infarcted myocardium with increased oxidative stress and collagen infiltration (19). TIIA can inhibit hydrogen peroxide-induced cardiomyocyte apoptosis (20). However, whether TIIA administration can reverse cardiac fibrosis in MI-induced HF rats via inhibiting oxidative stress is not known. In addition, it 
is not clear whether NADPH oxidase (Nox) 4 is involved in the oxidative stress in the fibrosis of cardiac fibroblasts (CFs) induced by Ang II. These questions were addressed by the present study.

\section{Materials and methods}

Animals.Experiments werecarried outon maleSprague-Dawley (SD) rats (age, 5-6 weeks; weight, 180-200 g; Vital River Biological Co., Ltd, Beijing, China). A total of 80 rats were used in the present study. All procedures were approved by the Experimental Animal Care and Use Committee of Nanjing University of Chinese Medicine (approval no. 17064512), and were conducted in accordance with the Guide for the Care and Use of Laboratory Animals (National Institutes of Health publication no. 85-23, revised in 1996; pubmed.ncbi.nlm.nih. gov/25121211/). The rats were kept in a temperature $\left(22 \pm 1^{\circ} \mathrm{C}\right)$ and humidity (30-60\%)-controlled room on a 12-h light/dark cycle with free access to standard chow and tap water. The rats were sacrificed using an overdose of sodium pentobarbital (100 mg/kg intravenously) for sample collection. Death was confirmed by the absence of heartbeat, corneal reflexes and paw withdrawal response to a noxious pinch.

Myocardial infarction model. The myocardial infarction (MI) in rats was induced by coronary artery ligation with sterile techniques as previously reported (21). Briefly, the rats were anesthetized with sodium pentobarbital $(50 \mathrm{mg} / \mathrm{kg}$, i.p.) and randomly subjected to the ligation of the left anterior descending (LAD) coronary artery or sham-operation. The heart was exposed through a left intercostal thoracotomy and the left coronary artery was looped by a single nylon suture (7-0). The LAD was ligated under $1-1.5 \mathrm{~mm}$ from the left atrial appendage in rats. The heart was then quickly repositioned into the chest. The sham rats were treated in the same way as the coronary ligation rats except that their coronary arteries were not ligated. After $24 \mathrm{~h}$, the rats were randomly assigned to four groups: i) Sham + saline group, ii) sham + TIIA group, iii) $\mathrm{MI}+$ saline group and iv) $\mathrm{MI}+$ TIIA group ( $\mathrm{n}=8$ for each group). TIIA (1.5 mg/kg/d/500 $\mu \mathrm{l}$, Sigma-Aldrich; Merck KGaA) (22) was orally gavaged to the rats in sham + TIIA and MI + TIIA groups for 28 days, while the rats in the other two groups received the same volume of saline. The rats were euthanized using an overdose of sodium pentobarbital (100 mg/kg intravenously) if malaise or surgical site putrescence presented.

Echocardiography. Transthoracic echocardiography was performed using an ultrasound system (VisualSonics Inc.) with a 21-MHz probe under isoflurane anesthesia (2.5\%) to measure the LV end-systolic diameter (LVESD), LV end-diastolic diameter (LVEDD), LV volumes in diastole (LVVd) and LV volumes in systole (LVVs). The LV ejection fraction(EF) and fractional shortening (FS) were calculated as $\mathrm{EF}=(\mathrm{LVVd}-\mathrm{LVVd}) / \mathrm{LVVd} \times 100$ and FS = (LVEDD-LVESD)/LVEDD x100. The measurements over three consecutive cardiac cycles were averaged.

Hemodynamic monitoring. Rats were anesthetized with sodium pentobarbital $(50 \mathrm{mg} / \mathrm{kg}$, i.p.). A conductance micromanometer catheter (1.4F, Millar Instruments, Inc.) was inserted into the LV chamber via the left carotid artery across the aortic valve. The left ventricular systolic pressure (LVSP) and LV end-diastolic pressure (LVEDP), maximum of the first differentiation of $\mathrm{LV}$ pressure $(\mathrm{LV}+\mathrm{dp} / \mathrm{dt})$ and decline (LV - dp/dt) were obtained with a PowerLab data acquisition system (ADInstruments Pty Ltd.).

Masson trichrome staining. The rats were sacrificed with an overdose of sodium pentobarbital (100 mg/kg, i.p.) and the hearts were harvested. Serial dehydration was performed using an ethanol concentration gradient, followed by paraffin embedding. Sections of the heart $(5 \mu \mathrm{m})$ were examined by Masson's trichrome staining according to the manufacturer's instructions (Wuhan Servicebio Technology Co., Ltd.) to determine the extent of fibrosis. Three to five random fields of view were selected in three sections from each animal for observation under a light microscope (Zeiss AG). Captured images were analyzed using Image-Pro Plus software 6.0 (Media Cybernetics, Inc.).

Culture of CFs. Rat CFs were isolated from 1-3 day-old SD rats (Beijing Vital River Laboratory Animal Technology Co., Ltd.). A total of 60 cubs were used in the present study. The hearts were collected following the pups anesthesia under isoflurane (3.5\%). Briefly, CFs were separated from cardiomyocytes by gravity separation and grown to confluence on $10 \mathrm{~cm}$ cell culture dishes with growth media [DMEM (Gibco; Thermo Fisher Scientific, Inc.] including 10\% FBS, $1 \%$ penicillin and $1 \%$ streptomycin) at $37^{\circ} \mathrm{C}$ in humid air with $5 \% \mathrm{CO}_{2}$ and $95 \% \mathrm{O}_{2}$. The CFs from the second passage were incubated with Ang II $\left(10^{-6} \mathrm{M}\right.$, Sigma-Aldrich; Merck KGaA) for $24 \mathrm{~h}$ to induce the fibrotic phenotype. The CFs were then assigned to four groups: i) PBS group, ii) TIIA $(10 \mu \mathrm{M})$ group, iii) Ang II group and iv) Ang II + TIIA $(10 \mu \mathrm{M})$ group.

Reverse transcription-quantitative PCR. The total RNA in $\mathrm{LV}$ or CFs $\left(1 \times 10^{6}\right.$ cells $\left./ \mathrm{ml}\right)$ was extracted with TRIzol ${ }^{\circledR}$ (Thermo Fisher Scientific, Inc.). cDNA was extracted from RNA with reverse transcription using $10 \mu \mathrm{l}$ random primers according to the instructions of the PrimeScript ${ }^{\mathrm{TM}}$ RT Master Mix (Takara Biotechnology Co., Ltd.) and stored at $-70^{\circ} \mathrm{C}$ before use. Collagen I, collagen III, $\alpha$-smooth muscle actin (SMA), TGF- $\beta$, matrix metalloproteinase (MMP) 2 and MMP9 mRNA levels were determined with Synergy Brands Green I fluorescence (Applied Biosystems) in accordance with the manufacturer's protocols. All samples were amplified in triplicates for 45 cycles in a 384 -well plate $\left(95^{\circ} \mathrm{C}\right.$ for $15 \mathrm{sec}$, then $60^{\circ} \mathrm{C}$ for $1 \mathrm{~min}$ ). The relative gene expression was determined by calculating the values of $\Delta^{\mathrm{Cq}}$ as a relative quantity to the endogenous control (23). These experiments were replicated three times. The primers sequences are listed in Table I.

Western blotting. CF samples were lysed in modified RIPA buffer (BioChannel Biological Technology Co., Ltd.). The protein concentration was determined using a BCA assay (Beyotime Institute of Biotechnology). A total of 30-50 $\mu \mathrm{g}$ of protein was separated using SDS-PAGE on $8 \%$ gels, then transferred to a PVDF membrane. The membrane was blocked with $5 \%$ skimmed milk powder at room temperature for $1 \mathrm{~h}$ 
Table I. List primers used for reverse transcription-quantitative PCR.

\begin{tabular}{lcll}
\hline Gene & Species & \multicolumn{1}{c}{ Forward primer, 5'-3' } & \multicolumn{1}{c}{ Reverse primer, 5'-3' } \\
\hline Collagen I & Rat & TCAAGATGGTGGCCGTTAC & CTGCGGATGTTCTCAATCTG \\
Collagen III & Rat & CGAGATTAAAGCAAGAGGAA & GAGGCTTCTTTACATACCAC \\
TGF- $\beta$ & Rat & CAGGGAGTAAGGGACACGA & ACAGCAGTTAGGAACCCAGAT \\
$\alpha-S M A$ & Rat & GTCCCAGACATCAGGGAGTAA & TCGGATACTTCAGCGTCAGGA \\
MMP2 & Rat & CCCCATGTGTCTTCCCCTTC & AGCTCCTGGATCCCCTTGAT \\
MMP9 & Rat & AGGGCCCCTTTCTTATTGCC & CGAGTAACGCTCTGGGGATC \\
GAPDH & Rat & GGCACAGTCAAGGCTGAGAATG & ATGGTGGTGAAGACGCCAGTA
\end{tabular}

$\alpha$-SMA, $\alpha$-smooth muscle actin; MMP, matrix metalloproteinase.

and probed overnight at $4^{\circ} \mathrm{C}$ with primary antibodies against collagen I (1:1,000; cat. no. ab254113; Abcam), collagen III (1:5,000; cat. no. ab7778; Abcam), TGF- $\beta(1: 1,000$; cat.no. ab215715; Abcam), $\alpha$-SMA(1:10,000; cat. no. ab124964; Abcam), MMP2 (1:2,000; cat. no. ab92536; Abcam) and MMP9 (1:1,000; cat. no. ab228402; Abcam) primary antibodies (Abcam), followed by incubation with a HRP-conjugated goat anti-rabbit secondary antibody (1:10,000, cat. no. ab7090; Abcam) at $37^{\circ} \mathrm{C}$ for $1 \mathrm{~h}$. The bands were visualized using the enhanced chemiluminescence (ECL) substrate (BioChannel Biological Technology Co., Ltd.). The total AT1R protein level was normalized to the protein level of GAPDH (Bioworld Technology Inc.). Images were analyzed using Image-Pro Plus software (version 6.0; XRayScan; CAD/CAM Services, Inc.).

Nox4 overexpression. Recombinant adenoviral vectors harboring green fluorescent protein (Ad-GFP) and Nox4 (Ad-Nox4) were constructed and packaged by Shanghai GeneChem Co., Ltd. In the in vivo experiment, adenovirus (200 $\mu \mathrm{l} / \mathrm{rat}, 1 \times 10^{12}$ plaque-forming units $/ \mathrm{ml}$ ) was injected into the rats via the tail vein. In the in vitro experiment, $20 \mu \mathrm{l}$ original solution was diluted in $2 \mathrm{ml}$ Enhance Infection Solution (Shanghai GeneChem Co., Ltd.). The CFs were transfected with serum-free medium containing Ad-Nox4 or Ad-GFP at $37^{\circ} \mathrm{C}$ for $24 \mathrm{~h}$.

Superoxide dismutase (SOD) activity level. The rats were sacrificed with an overdose of pentobarbital (100 mg/kg, i.p.). The LV samples were collected and homogenized in lysis buffer (Thermo Fisher Scientific, Inc.). SOD was measured using a commercial kit (Nanjing Jiancheng Bioengineering Institute; cat. no. A001-3-2) according to the manufacturer's instructions using a microplate reader (BioTek Instruments, Inc.).

Malondialdehyde level in the heart. After homogenizing the LV samples, malondialdehyde (MDA) levels in the LV were determined using an ELISA kit (Wuhan USCN Business Co., Ltd.; cat. no. CEA597Ge) following the manufacturer's instructions.

Measurement of Nox activity. The Nox activity in the heart was measured by enhanced lucigenin chemiluminescence. Briefly, NADPH $(100 \mu \mathrm{M})$ was added to the media as a substrate to react with Nox and generate superoxide anions. The light emission produced by the reaction of lucigenin $(5 \mu \mathrm{M})$ with superoxide anions was measured with a microplate reader (BioTek Instruments, Inc.) once every minute for $10 \mathrm{~min}$. The values representing Nox activity were expressed as the mean light units (MLU) per min per mg of protein.

Measurement of superoxide anions. The level of superoxide anions in the heart was determined by lucigenin-derived chemiluminescence. Briefly, the reaction with superoxide anions was started by adding dark-adapted lucigenin $(5 \mu \mathrm{M})$ to each sample to cause photon emission, which was measured with a microplate reader (BioTek Instruments, Inc.) once every minute for $10 \mathrm{~min}$. The values representing the superoxide anions level were expressed as the MLU per min per mg of protein.

Statistical analysis. Data are presented as the mean \pm standard error of the mean and were analyzed using GraphPad Prism 7.0 (GraphPad software Inc.). Statistics were completed using one-way ANOVA, followed by Bonferroni test for post hoc analysis when multiple comparisons were made. $\mathrm{P}<0.05$ was considered to indicate a statistically significant difference.

\section{Results}

TIIA improves cardiac function of rats with MI-induced $H F$. EF, FS, LVSP and $\mathrm{LV} \pm \mathrm{dp} / \mathrm{dt}_{\max }$ were reduced in rats with MI-induced $\mathrm{HF}$ and this reduction was reversed by TIIA treatment. LVVs, LVVd, LVESD, LVEDD and LVEDP were increased in MI-induced HF rats and this increase was attenuated by TIIA treatment (Fig. 1).

TIIA attenuates LV fibrosis in rats with MI-induced HF. The mRNA expression levels of collagen I, collagen III, TGF- $\beta$, $\alpha$-SMA, MMP2 and MMP9 in LV of MI rats were increased compared with sham rats. The increased levels of collagen I, collagen III, TGF- $\beta, \alpha$-SMA, MMP 2 and MMP9 in the LVs of MI rats were inhibited after TIIA administration (Fig. 2).

TIIA attenuates Ang II-induced fibrosis of CFs. The mRNA expression levels of collagen I, collagen III, TGF- $\beta, \alpha$-SMA, MMP2 and MMP9 increased in Ang II-treated CFs and this increase was reversed after TIIA treatment (Fig. 3A). 

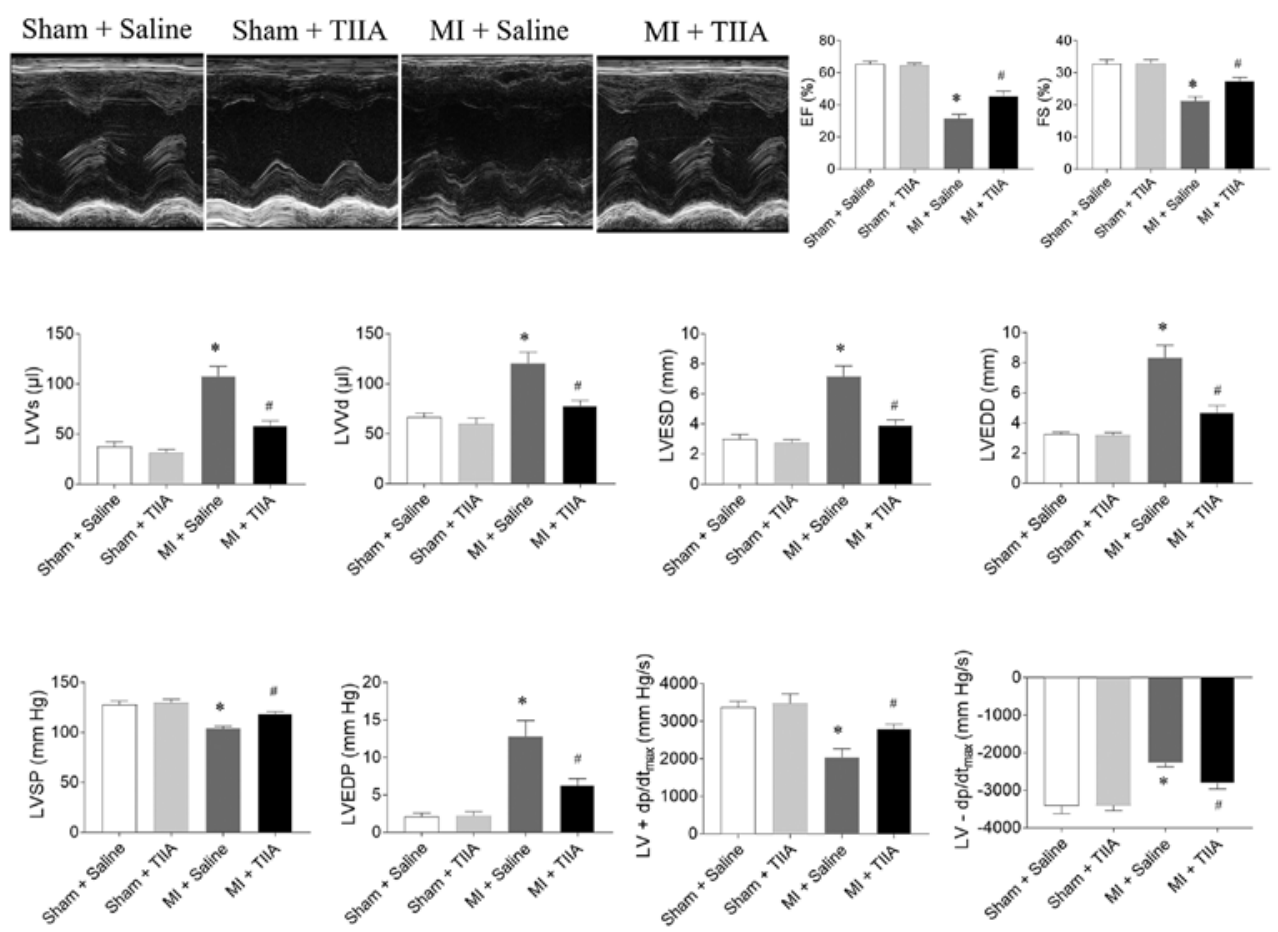

Figure 1. TIIA improves cardiac dysfunction in rats with MI-induced heart failure. TIIA treatment improved the decreases of LV EF, FS, LVSP and the $\mathrm{LV} \pm \mathrm{dp} / \mathrm{dt}_{\max }$ and the increases of LVVS, LVVD, LVESD and LVEDD in MI rats. The results are expressed as mean \pm standard error of the mean. $\mathrm{n}=8$. ${ }^{*} \mathrm{P}<0.05$ vs. the Sham + Saline group; ${ }^{~} \mathrm{P}<0.05$ vs. the MI + Saline group. TIIA, tanshinone IIA; MI, myocardial infarction; LV, left ventricular; EF, ejection fraction; FS, fractional shortening; LVSP, LV systolic pressure; $\mathrm{LV} \pm \mathrm{dp} / \mathrm{dt}_{\max }$, maximum of the first differentiation of LV pressure; LVVS, LV volume in systole; LVVD, LV volume in diastole; LVESD, LV end-systolic diameter; LVEDD, LV end-diastolic diameter.

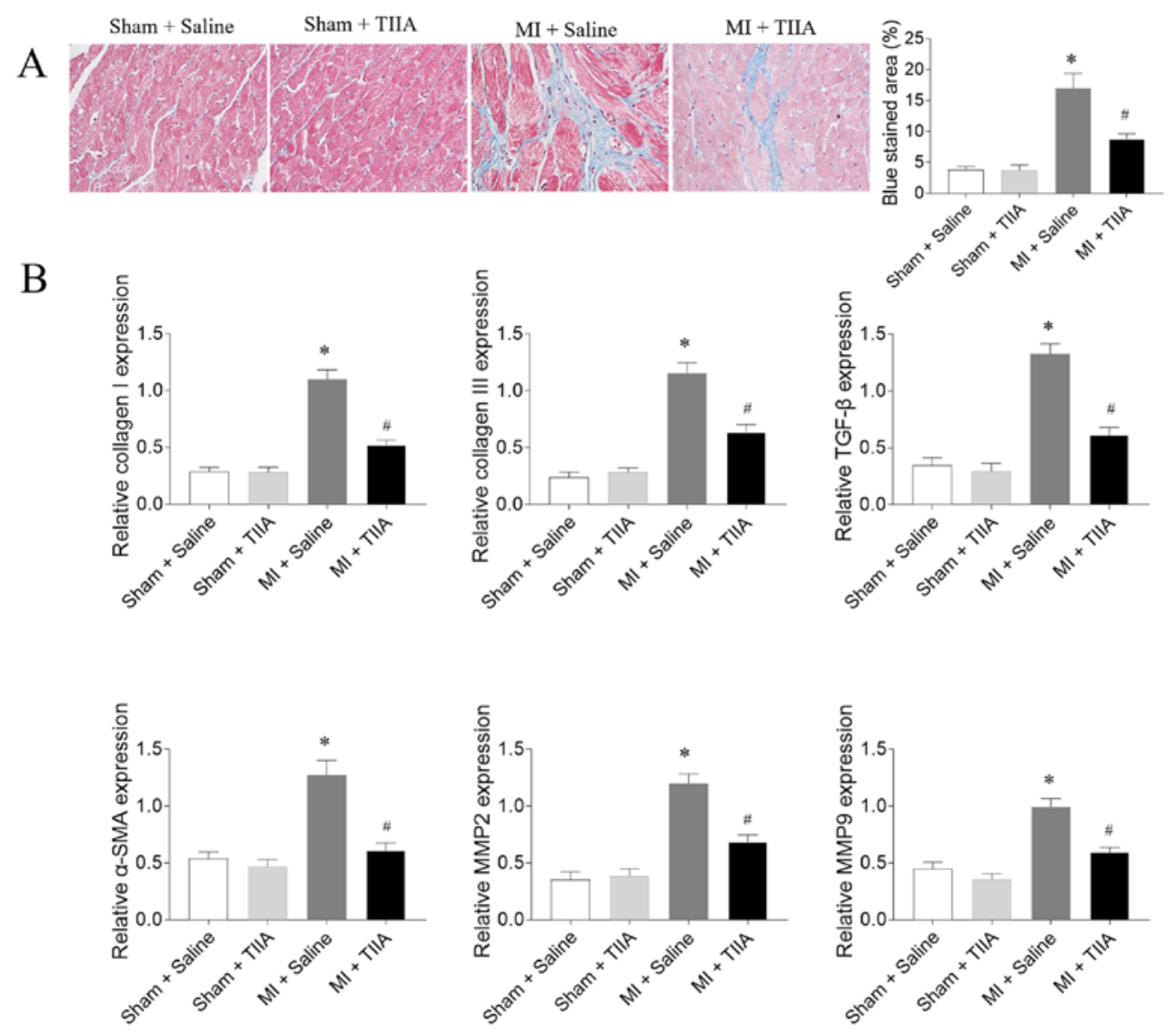

Figure 2. TIIA attenuates LV fibrosis in rats with MI-induced heart failure. (A) TIIA treatment attenuated LV fibrosis in MI rats, the blue area represents the fibrosis. Magnification, $x 400$. (B) TIIA treatment inhibited the increases of collagen I, collagen III, SMA, TGF- $\beta$, MMP2 and MMP9 in MI rats. The results are expressed as mean \pm standard error of the mean. $n=8$. ${ }^{\mathrm{P}}<0.05$ vs. the Sham + Saline group; ${ }^{*} \mathrm{P}<0.05$ vs. the MI + Saline group. TIIA, tanshinone IIA; LV, left ventricular; MI, myocardial infarction; SMA, $\alpha$-smooth muscle actin; MMP, matrix metalloproteinase. 
A
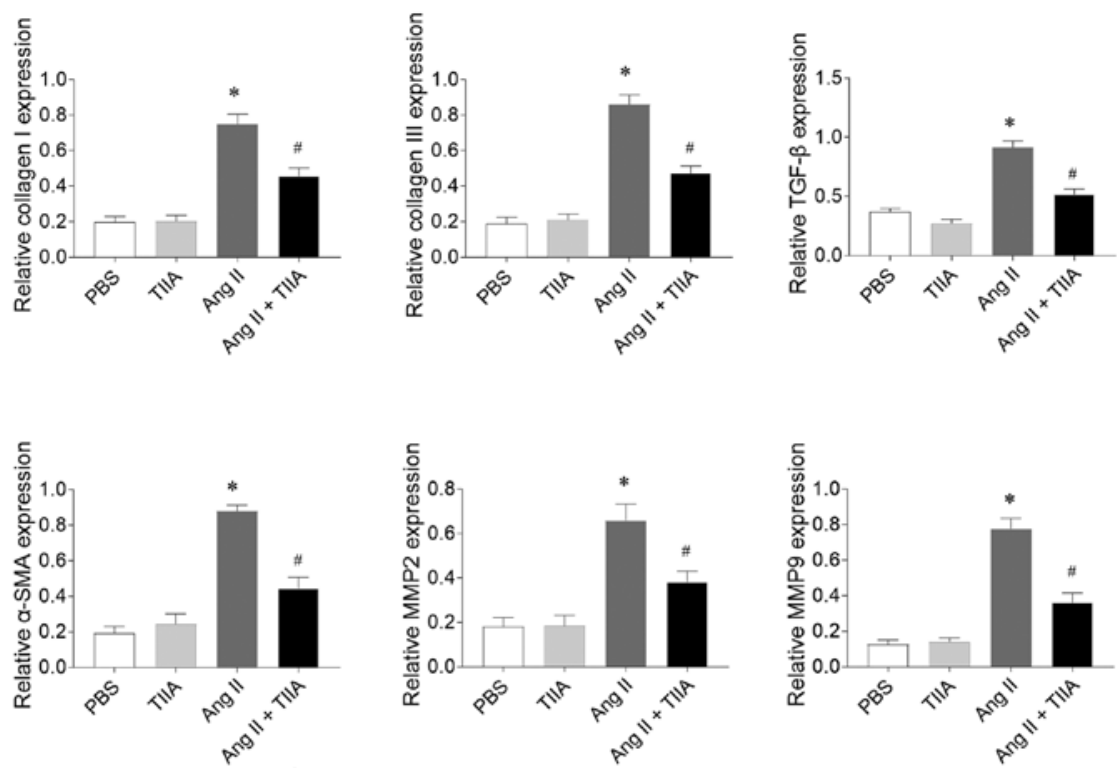
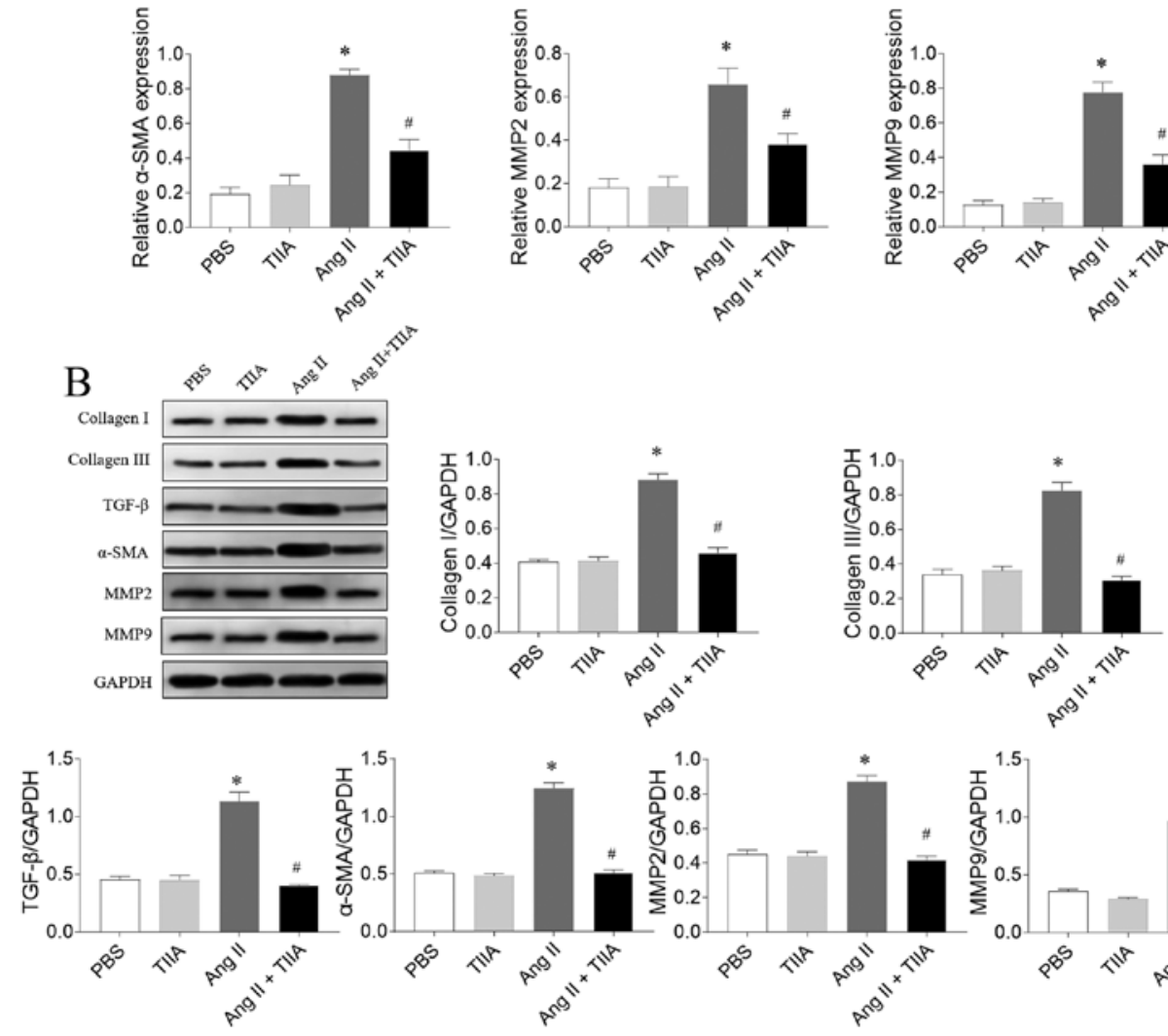

Figure 3. TIIA attenuates CFs fibrosis induced by Ang II. (A) TIIA treatment inhibited the increased mRNA expression levels of collagen I, collagen III, SMA, TGF- $\beta$, MMP2 and MMP9 in Ang II-treated CFs. (B) TIIA treatment inhibited the increased protein expression of collagen I, collagen III, $\alpha$-SMA, TGF- $\beta$, MMP2 and MMP9 in Ang II-treated CFs. The results are expressed as mean $\pm \mathrm{SE}$. ${ }^{*} \mathrm{P}<0.05$ vs. the PBS group; ${ }^{\#} \mathrm{P}<0.05$ vs. the Ang II group. TIIA, tanshinone IIA; CFs, cardiac fibroblasts; Ang II, angiotensin II; SMA, $\alpha$-smooth muscle actin; MMP, matrix metalloproteinase.

The Ang II-induced increases in protein expression levels of collagen I, collagen III, TGF- $\beta, \alpha$-SMA, MMP2 and MMP9 in CFs were inhibited after TIIA treatment (Fig. 3B).

TIIA attenuates oxidative stress in the LVs of HF rats and Ang II-treated CFs. SOD activity level was reduced in the LVs of MI-induced HF rats and this reduction was reversed by TIIA. MDA, superoxide anions and Nox activity levels were significantly increased in LV of MI-induced HF rats and this increase was inhibited after TIIA treatment (Fig. 4A). The decrease of SOD activity and the increases of MDA, superoxide anions and Nox activity levels in Ang II-treated CFs were reversed by TIIA administration (Fig. 4B).

Nox4 overexpression reverses the improving effects of TIIA on cardiac function in $\mathrm{HF}$ rats. The expression level of Nox was increased in the heart rats treated with Ad-Nox4 (Fig. 5A). The effects of TIIA in improving $\mathrm{LV} \pm \mathrm{dp} / \mathrm{dt}_{\max }, \mathrm{EF}, \mathrm{FS}$ and LVSP in MI-induced HF rats were reversed by Nox4 overexpression. Moreover, Nox4 overexpression also reversed the effects of TIIA in decreasing LVVs, LVVd, LVESD, LVEDD and LVEDP in MI-induced HF rats (Fig. 5B and C.).

Nox4 overexpression reverses the inhibitory effects of TIIA on $L V$ fibrosis in HF rats. Nox4 overexpression reversed the inhibiting effects of TIIA on the increases in the mRNA expression levels of collagen I, collagen III, TGF- $\beta, \alpha$-SMA, MMP 2 and MMP9 in the LVs of rats with MI-induced HF (Fig. 6).

Nox4 overexpression reverses the inhibitory effects of TIIA on Ang II-induced CF fibrosis. Nox4 overexpression reversed the inhibitory effects of TIIA on Ang II-induced increases in the mRNA expression levels of collagen I, collagen III, TGF- $\beta$, $\alpha$-SMA, MMP2 and MMP9 in CFs (Fig. 7). 
A
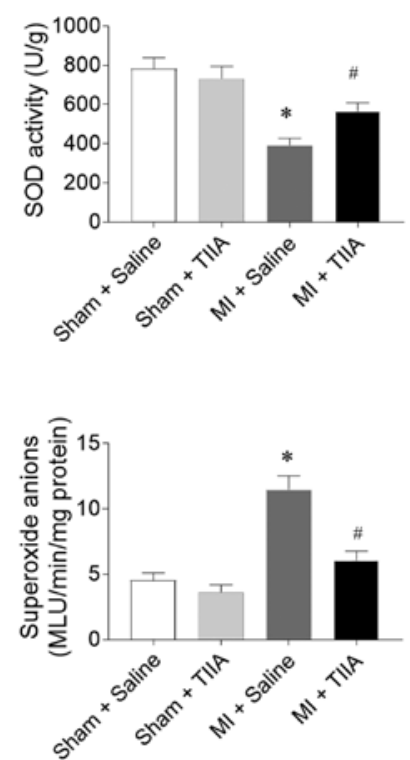

$\mathrm{B}$
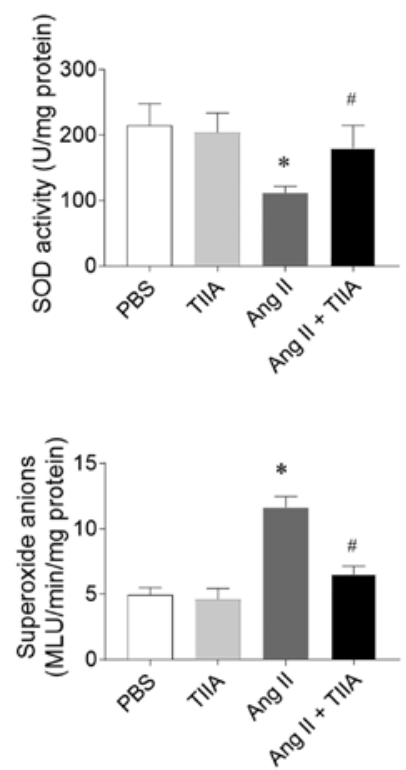
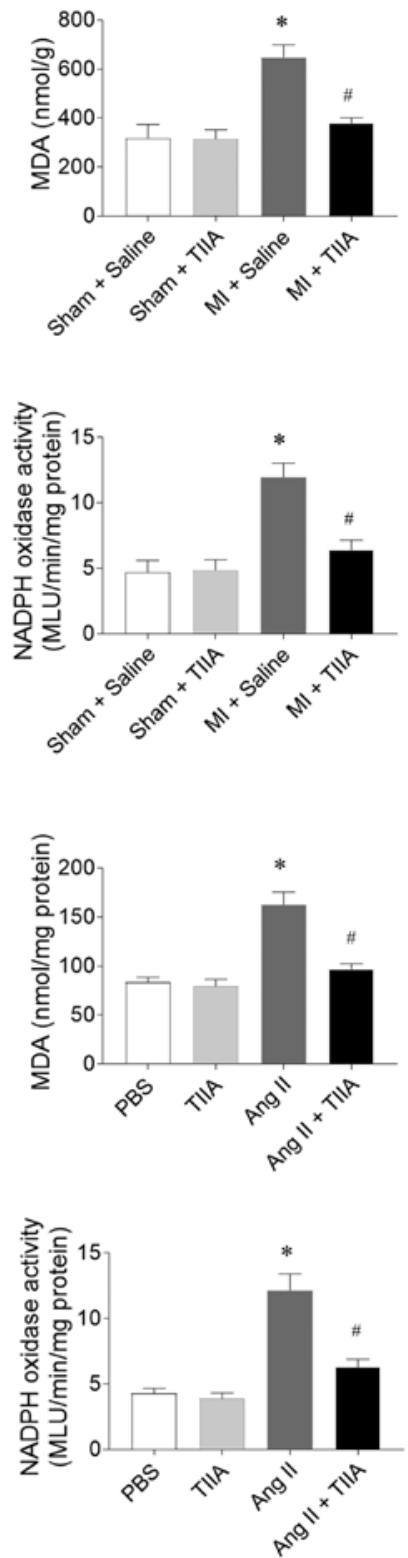

Figure 4. TIIA attenuates the increase of oxidative stress in the heart of myocardial infarction MI rats and the Ang II-treated CFs. (A) TIIA treatment increased the decreased SOD activity and inhibited the increases of MDA, superoxide anions and NADPH oxidase activity in the LV of MI rats. (B) TIIA treatment increased the decrease of SOD activity and inhibited the increases of MDA, superoxide anions and NADPH oxidase activity in Ang II-treated CFs. The results are expressed as mean \pm standard error of the mean. $n=8$. ${ }^{*} \mathrm{P}<0.05$ vs. the (A) Sham + Saline or (B) PBS group; ${ }^{\#} \mathrm{P}<0.05$ vs. the (A) MI + Saline or (B) Ang II group. TIIA, tanshinone IIA; MI, myocardial infarction; Ang II, angiotensin II; CFs, cardiac fibroblasts; SOD, superoxide dismutase; MDA, malondialdehyde; LV, left ventricular.

\section{Discussion}

The present study found that TIIA improved cardiac dysfunction in the rats with MI-induced HF. The fibrosis of LV in HF rats and Ang II-treated CFs was ameliorated following TIIA administration. Furthermore, oxidative stress was enhanced in LV of HF rats and in Ang II-treated CFs, as indicated by the decrease of SOD activity and the increases of MDA, superoxide anions and Nox activity levels, all reversed by TIIA treatment. Nox4 overexpression inhibited the effects of TIIA in improving cardiac dysfunction in HF rats and the fibrosis of LV in HF rats and Ang II-treated CFs.

The results of the present study demonstrated that TIIA treatment ameliorated the decreases of EF, FS, LVSP and $\mathrm{LV} \pm \mathrm{dp} / \mathrm{dt}_{\max }$ and the increases of LVVs, LVVd, LVESD,
LVEDD and LVEDP in MI-induced HF rats, indicating that TIIA improved cardiac dysfunction in HF. This finding is consistent with a previous finding that TIIA could significantly improve heart function in left anterior descending (LAD) ligation-induced HF (22).

As a complex pathological process involving myocardial fibrosis, cardiac hypertrophy and cardiomyocyte apoptosis, LV remodeling is often caused by cardiovascular diseases (CVDs) such as HF, hypertension, or myocardial infarction $(24,25)$. Cardiac fibrosis is a significant global health problem caused by pathological stimuli to the heart. Previous studies demonstrated that TIIA could inhibit the proliferation of mouse cardiac fibroblasts in cultures by MTT assay (26) and attenuate high glucose-mediated collagen synthesis through inhibiting the TGF- $\beta 1 /$ Smad signaling pathway in cardiac 
A

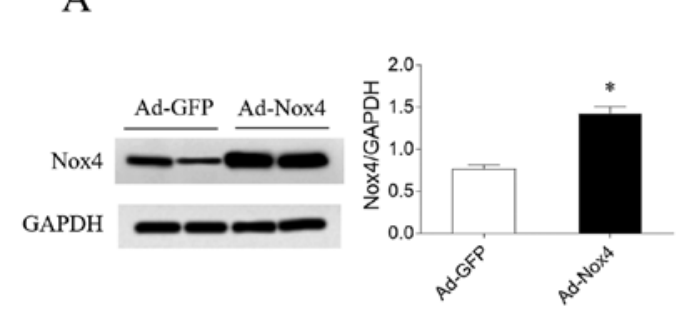

$\mathrm{C}$
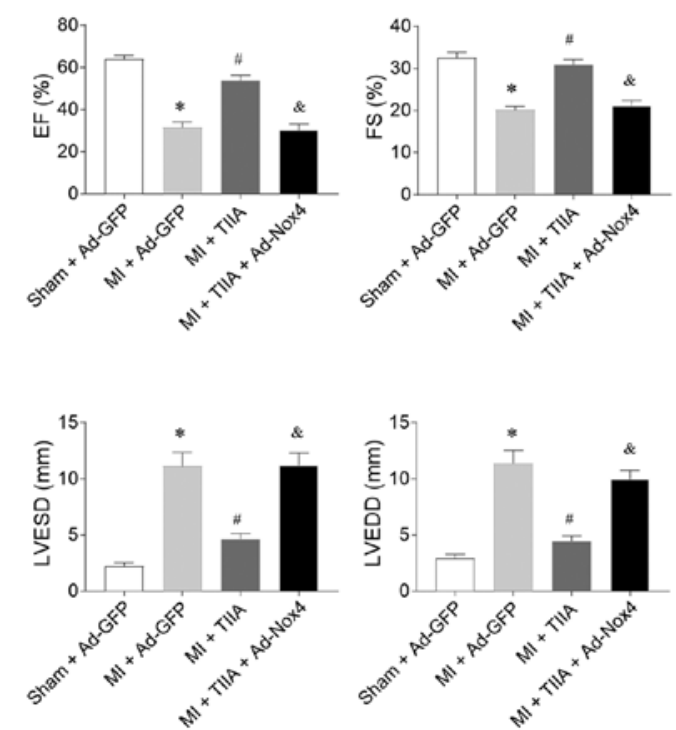

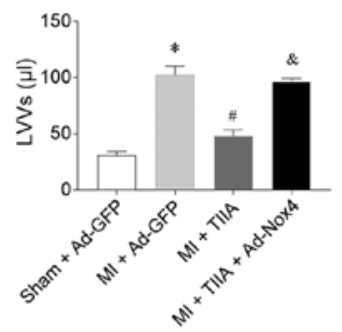

$\mathrm{B}$
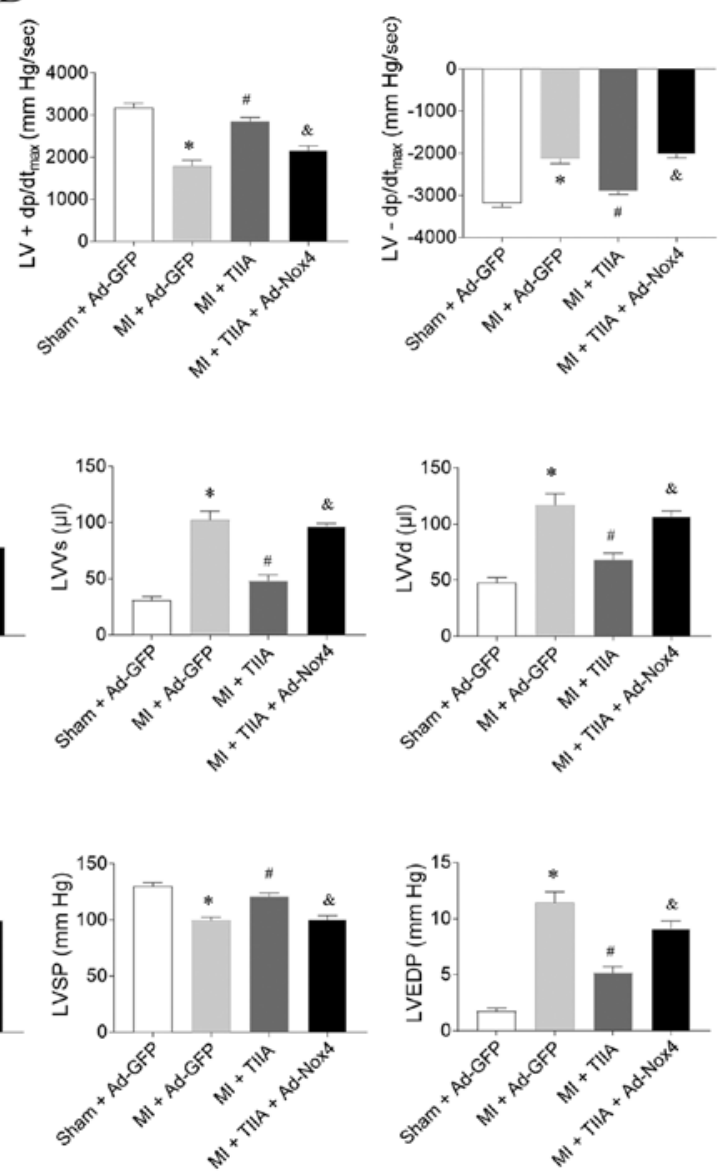

Figure 5. Nox4 overexpression reverses the effects of TIIA in improving cardiac dysfunction on rats with MI-induced heart failure. (A) The expression level of Nox4 was increased in the heart rats treated with adenoviral (Ad)-Nox4. (B) Nox4 overexpression reversed the improving effects of TIIA on the decreases of LV $\pm \mathrm{dp} / \mathrm{dt}_{\max }$ in MI rats. (C) Nox4 overexpression reversed the improving effects of TIIA on the decreases of LV EF, FS and LVSP and the increases of LVVS, LVVD, LVESD and LVEDD in MI rats. The results are expressed as mean \pm standard error of the mean. $\mathrm{n}=8$. ${ }^{*} \mathrm{P}<0.05$ vs. the (A) Ad-GFP or (B and C) Sham + Ad-GFP group; ${ }^{\#} \mathrm{P}<0.05$ vs. the MI + Ad-GFP group; ${ }^{\circledR} \mathrm{P}<0.05$ vs. the MI + TIIA group. Nox, NADPH oxidase; TIIA, tanshinone IIA; $\mathrm{MI}$, myocardial infarction; $\mathrm{LV} \pm \mathrm{dp} / \mathrm{dt}_{\max }$, maximum of the first differentiation of $\mathrm{LV}$ pressure; $\mathrm{LV}$, left ventricular; $\mathrm{EF}$, ejection fraction; $\mathrm{FS}$, fractional shortening; LVSP, LV systolic pressure; LVVS, LV volume in systole; LVVD, LV volume in diastole; LVESD, LV end-systolic diameter; LVEDD, LV end-diastolic diameter.
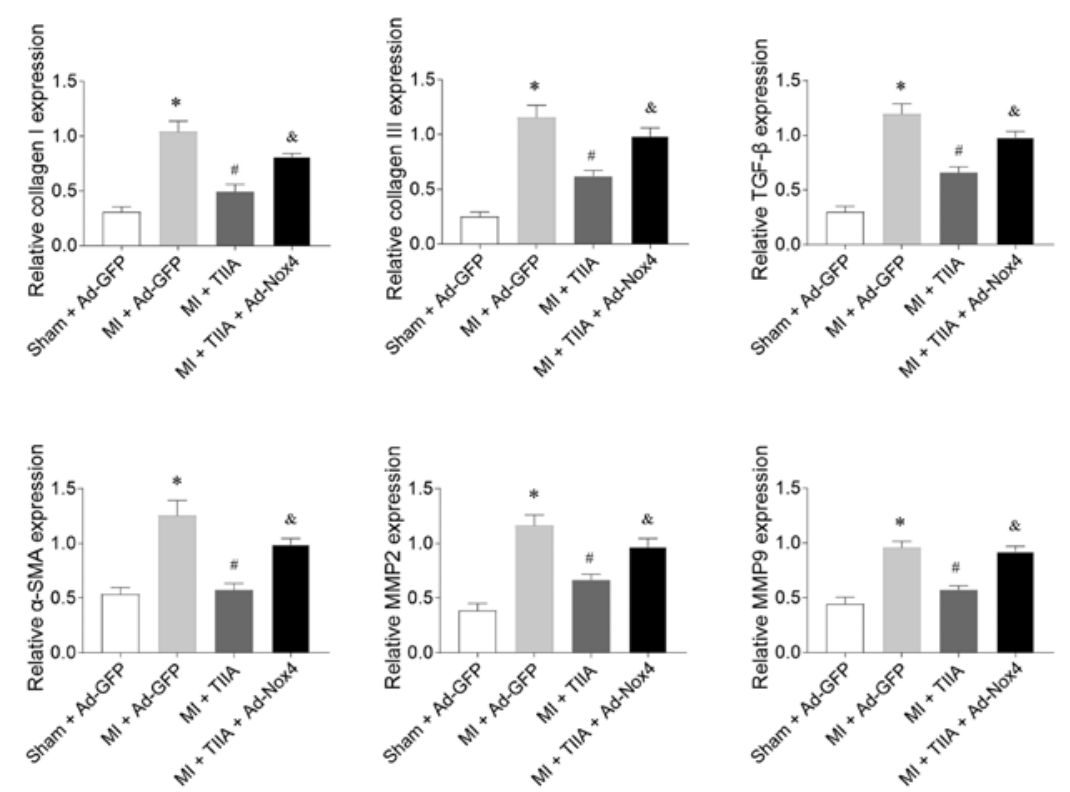

Figure 6. Nox4 overexpression reverses the effects of TIIA in inhibiting LV fibrosis in rats with MI-induced heart failure. Nox4 overexpression reversed the effects of TIIA in attenuating the increases of collagen I, collagen III, SMA, TGF- $\beta$, MMP2 and MMP9 in the LV of MI rats. The results are expressed as mean \pm standard error of the mean. $n=8 .{ }^{~} \mathrm{P}<0.05$ vs. the Sham + Ad-GFP group; ${ }^{*} \mathrm{P}<0.05$ vs. the MI + Ad-GFP group; ${ }^{\&} \mathrm{P}<0.05$ vs. the MI + TIIA group. Nox, NADPH oxidase; TIIA, tanshinone IIA; LV, left ventricular; MI, myocardial infarction; SMA, $\alpha$-smooth muscle actin; MMP, matrix metalloproteinase. 

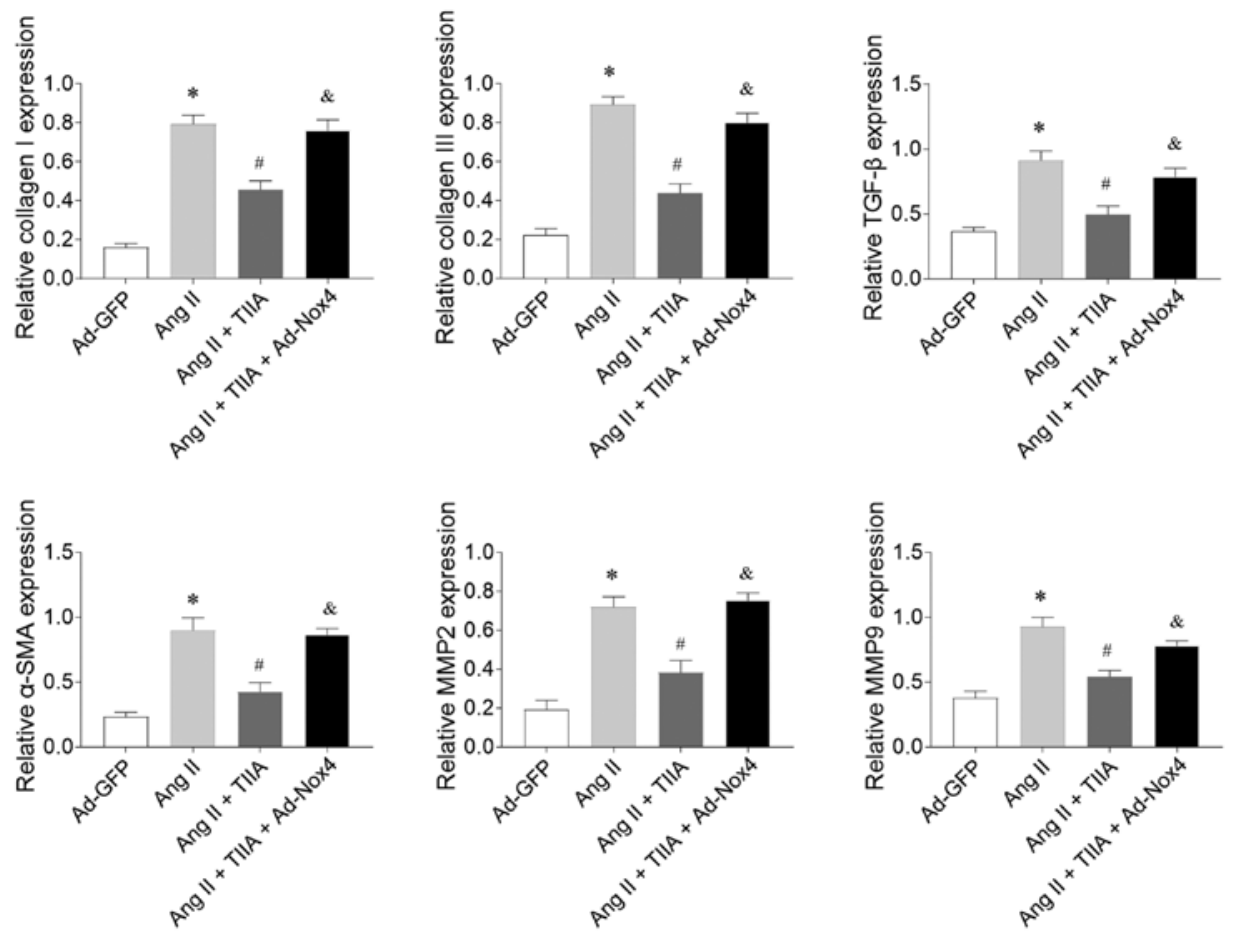

Figure 7. Nox4 overexpression reversed the inhibiting effects of TIIA on Ang II-induced fibrosis of CFs. Nox4 overexpression reversed the effects of TIIA in attenuating the increases of collagen I, collagen III, SMA, TGF- $\beta$, MMP2 and MMP9 in the Ang II-treated CFs. The results are expressed as mean \pm standard error of the mean. "P<0.05 vs. the Ad-GFP group; ${ }^{~} \mathrm{P}<0.05$ vs. the Ang II group; ${ }^{\circledR} \mathrm{P}<0.05$ vs. the Ang II + TIIA group. Nox, NADPH oxidase; TIIA, tanshinone IIA; Ang II, angiotensin II; CFs, cardiac fibroblasts; SMA, $\alpha$-smooth muscle actin; MMP, matrix metalloproteinase.

fibroblasts (27). The present study found that the increases of collagen I, collagen III, TGF- $\beta, \alpha$-SMA, MMP2 and MMP9 in LV of MI-induced HF rats were abolished following TIIA administration. In addition, TIIA treatment inhibited the increases of collagen I, collagen III, TGF- $\beta, \alpha$-SMA, MMP2 and MMP9 in Ang II-treated CFs. These results suggested that TIIA attenuated the fibrosis of LV in HF.

As important molecules in the living organisms, reactive oxygen species (ROS) are involved in many signaling pathways (28). However, the overproduction of ROS serves a significant role in the development of CVDs $(29,30)$. Oxidative stress, a key contributor to organ damage, is associated with various diseases (31), including cardiac fibrosis (32). Sodium TIIA sulfonate inhibits the increased production of ROS and expression of TGF- $\beta 1$ stimulated by Ang II in human atrial fibroblasts (33). TIIA significantly inhibits $\mathrm{H}_{2} \mathrm{O}_{2}$-induced collagen synthesis via attenuating Nox activity and ROS generation (34). Due to its antioxidative property, TIIA protects two-kidney, two-clip hypertensive rats from cardiac dysfunction and fibrosis, partially via reducing Nox activity, but without changing blood pressure (35). In the present study, the results demonstrated that SOD activity was significantly reduced and that the MDA, superoxide anions and Nox activity levels were increased in LV of HF rats, then decreased by TIIA treatment. Furthermore, the decrease of SOD activity and the increases of MDA, superoxide anions and Nox activity in Ang II-treated CFs were reversed by TIIA administration. These results indicated that in the heart of MI-induced HF rats, the oxidants and antioxidants were imbalanced, which could be reversed by TIIA; TIIA could curb cardiac fibrosis in HF via inhibiting oxidative stress.
TIIA might be a potent agent against the fibrosis in the hearts of lipopolysaccharide-treated mice partially via inhibiting Nox2 (36). The present study found that Nox4 overexpression reversed the effects of TIIA in ameliorating cardiac dysfunction in HF rats. The effects of TIIA in reducing collagen I, collagen III, TGF- $\beta, \alpha$-SMA, MMP2 and MMP9 on LV of HF rats were inhibited following Nox4 overexpression. These results demonstrated that Nox4 could regulate the inhibitory effects of TIIA on HF and cardiac fibrosis.

In addition to the oxidative stress explored in the present study, TIIA is involved in various other signal pathways related to cardiac diseases. TIIA protects cardiomyocytes and improves cardiac function by activating the AMP-activated protein kinase-mammalian target of rapamycin signaling pathway to inhibit apoptosis and induce autophagy (22). TIIA prevents LV remodeling of MI rats, mainly through repressing the Toll-like receptor 4/Myeloid differentiation primary response 88/NF- $\mathrm{BB}$ signaling pathway (37). A previous study demonstrated that TIIA can activate the phosphatidylinositol 3-kinase/protein kinase B/mTOR signaling pathway to relieve myocardial ischemia reperfusion injury in rats (12).

In the present study, it was not clear how TIIA improved cardiac fibrosis of MI rats via inhibiting oxidative stress. Since only MI model-induced cardiac fibrosis was investigated in the present study, whether cardiac fibrosis in other models could also be attenuated by TIIA remains unknown.

In conclusion, oxidative stress was enhanced in the hearts of HF rats, then attenuated by TIIA. TIIA restored the imbalance between oxidant and antioxidant levels. TIIA, functioning as an antioxidant, could improve cardiac dysfunction and attenuate fibrosis in HF rats and Ang II-induced fibrosis 
in CFs. Nox4 regulated the inhibitory effects of TIIA in HF and cardiac fibrosis. It is hoped these results could provide evidence for the clinical application of TIIA in treating HF-related cardiac fibrosis.

\section{Acknowledgements}

Not applicable.

\section{Funding}

The present study was supported by the Key Discipline Construction Project of Jiangsu Province (grant no. js1303).

\section{Availability of data and materials}

The datasets used or analyzed during the present study are available from the corresponding author upon request.

\section{Authors' contributions}

RC and WC made substantial contributions to conception and design of the study, and were involved in drafting the manuscript or revising it critically for important intellectual content. XH acquired analysed and interpreted the data. QR made substantial contributions to conception and design of the study, and was involved in drafting and revising the manuscript. All authors read and approved the final version of the manuscript.

\section{Ethics approval and consent to participate}

The present study was approved by the Experimental Animal Care and Use Committee of Nanjing University of Chinese Medicine (approval no. 2018031811).

\section{Patient consent for publication}

Not applicable.

\section{Competing interests}

The authors declare that they have no competing interests

\section{References}

1. Bui AL, Horwich TB and Fonarow GC: Epidemiology and risk profile of heart failure. Nat Rev Cardiol 8: 30-41, 2011.

2. Vos T, Flaxman AD, Naghavi M,Lozano R, Michaud C, Ezzati M, Shibuya K, Salomon JA, Abdalla S, Aboyans V, et al: Years lived with disability (YLDs) for 1160 sequelae of 289 diseases and injuries 1990-2010: A systematic analysis for the Global Burden of Disease Study 2010. Lancet 380: 2163-2196, 2012.

3. Raso A, Dirkx E, Philippen LE, Fernandez-Celis A, De Majo F, Sampaio-Pinto V, Sansonetti M, Juni R, El Azzouzi H, Calore M, et al: Therapeutic Delivery of miR-148a Suppresses Ventricular Dilation in Heart Failure. Mol Ther 27: 584-599, 2019.

4. Timmis A, Townsend N, Gale C, Grobbee R, Maniadakis N, Flather M, Wilkins E, Wright L, Vos R, Bax J, et al; ESC Scientific Document Group: European Society of Cardiology: Cardiovascular Disease Statistics 2017. Eur Heart J 39: 508-579, 2018.

5. Liu C, Yang CX, Chen XR, Liu BX, Li Y, Wang XZ, Sun W, Li $\mathrm{P}$ and Kong XQ: Alamandine attenuates hypertension and cardiac hypertrophy in hypertensive rats. Amino Acids 50: 1071-1081, 2018
6. Wang L, Liu C, Chen X and Li P: Alamandine attenuates long term hypertension induced cardiac fibrosis independent of blood pressure. Mol Med Rep 19: 4553-4560, 2019.

7. Yue Y, Meng K, Pu Y and Zhang X: Transforming growth factor beta (TGF- $\beta$ ) mediates cardiac fibrosis and induces diabetic cardiomyopathy. Diabetes Res Clin Pract 133: 124-130, 2017.

8. Yuan X, Pan J, Wen L, Gong B, Li J, Gao H, Tan W, Liang S, Zhang $\mathrm{H}$ and Wang X: miR-144-3p Enhances Cardiac Fibrosis After Myocardial Infarction by Targeting PTEN. Front Cell Dev Biol 7: 249, 2019.

9. Rai R, Sun T, Ramirez V, Lux E, Eren M, Vaughan DE and Ghosh AK: Acetyltransferase p300 inhibitor reverses hypertension-induced cardiac fibrosis. J Cell Mol Med 23: 3026-3031, 2019.

10. Tan CY, Wong JX, Chan PS, Tan H, Liao D, Chen W, Tan LW, Ackers-Johnson M, Wakimoto H, Seidman JG, et al: Yin Yang 1 Suppresses Dilated Cardiomyopathy and Cardiac Fibrosis Through Regulation of Bmp7 and Ctgf. Circ Res 125: 834-846, 2019.

11. Xu QQ, Xu YJ, Yang C, Tang Y, Li L, Cai HB, Hou BN, Chen HF, Wang Q, Shi XG, et al: Sodium Tanshinone IIA Sulfonate Attenuates Scopolamine-Induced Cognitive Dysfunctions via Improving Cholinergic System. BioMed Res Int 2016: 9852536, 2016.

12. Li Q, Shen L, Wang Z, Jiang HP and Liu LX: Tanshinone IIA protects against myocardial ischemia reperfusion injury by activating the PI3K/Akt/mTOR signaling pathway. Biomed Pharmacother 84: 106-114, 2016.

13. Wang X, Wei Y, Yuan S, Liu G, Lu Y, Zhang J and Wang W: Potential anticancer activity of tanshinone IIA against human breast cancer. Int J Cancer 116: 799-807, 2005.

14. Li C, Han X, Zhang H, Wu J and Li B: The interplay between autophagy and apoptosis induced by tanshinone IIA in prostate cancer cells. Tumour Biol 37: 7667-7674, 2016.

15. Gao S,Liu Z,LiH,LittlePJ,Liu P and Xu S: Cardiovascular actions and therapeutic potential of tanshinone IIA. Atherosclerosis 220: 3-10, 2012.

16. Chen FY, Guo R and Zhang BK: Advances in cardiovascular effects of tanshinone II(A). Zhongguo Zhong Yao Za Zhi 40: 1649-1653, 2015 (In Chinese).

17. Sag CM, Santos CX and Shah AM: Redox regulation of cardiac hypertrophy. J Mol Cell Cardiol 73: 103-111, 2014.

18. Madamanchi NR and Runge MS: Redox signaling in cardiovascular health and disease. Free Radic Biol Med 61: 473-501, 2013.

19. Bonnefont-Rousselot D, Mahmoudi A, Mougenot N, Varoquaux O, Le Nahour G, Fouret P and Lechat P: Catecholamine effects on cardiac remodelling, oxidative stress and fibrosis in experimental heart failure. Redox Rep 7: 145-151, 2002.

20. Yan SH, Zhao NW, Geng ZR, Shen JY, Liu FM, Yan D, Zhou J, Nie C, Huang CC and Fang ZY: Modulations of Keap1-Nrf2 signaling axis by TIIA ameliorated the oxidative stress-induced myocardial apoptosis. Free Radic Biol Med 115: 191-201, 2018.

21. Gan XB, Duan YC, Xiong XQ, Li P, Cui BP, Gao XY and Zhu GQ: Inhibition of cardiac sympathetic afferent reflex and sympathetic activity by baroreceptor and vagal afferent inputs in chronic heart failure. PLoS One 6: e25784, 2011.

22. Zhang X, Wang Q, Wang X, Chen X, Shao M, Zhang Q, Guo D, $\mathrm{Wu} \mathrm{Y,} \mathrm{Li} \mathrm{C,} \mathrm{Wang} \mathrm{W,} \mathrm{et} \mathrm{al:} \mathrm{Tanshinone} \mathrm{IIA} \mathrm{protects} \mathrm{against} \mathrm{heart}$ failure post-myocardial infarction via AMPKs/mTOR-dependent autophagy pathway. Biomed Pharmacother 112: 108599, 2019.

23. Livak KJ and Schmittgen TD: Analysis of relative gene expression data using real-time quantitative PCR and the 2(-Delta Delta C(T)) Method. Methods 25: 402-408, 2001

24. Koshman YE, Patel N, Chu M, Iyengar R, Kim T, Ersahin C, Lewis W, Heroux A and Samarel AM: Regulation of connective tissue growth factor gene expression and fibrosis in human heart failure. J Card Fail 19: 283-294, 2013.

25. Hou L, Guo J, Xu F, Weng X, Yue W and Ge J: Cardiomyocyte dimethylarginine dimethylaminohydrolase1 attenuates left-ventricular remodeling after acute myocardial infarction: Involvement in oxidative stress and apoptosis. Basic Res Cardiol 113: 28, 2018

26. Zhang Y, Zhang S and Chen X: Tanshinone IIA protects against cardiac fibrosis through inhibition of $\beta$-tubulin expression. J Biol Regul Homeost Agents 32: 1451-1455, 2018.

27. Tsai YT, Loh SH, Lee CY, Lee SP, Chen YL, Cheng TH and Tsai CS: Tanshinone IIA Inhibits High Glucose-Induced Collagen Synthesis via Nuclear Factor Erythroid 2-Related Factor 2 in Cardiac Fibroblasts. Cell Physiol Biochem 51: 2250-2261, 2018. 
28. Kura B, Szeiffova Bacova B, Kalocayova B, Sykora M and Slezak J: Oxidative Stress-Responsive MicroRNAs in Heart Injury. Int J Mol Sci 21: 358, 2020

29. Kim H, Yun J and Kwon SM: Therapeutic Strategies for Oxidative Stress-Related Cardiovascular Diseases: Removal of Excess Reactive Oxygen Species in Adult Stem Cells. Oxid Med Cell Longev 2016: 2483163, 2016.

30. Snezhkina AV, Kudryavtseva AV, Kardymon OL, Savvateeva MV, Melnikova NV, Krasnov GS and Dmitriev AA: ROS Generation and Antioxidant Defense Systems in Normal and Malignant Cells. Oxid Med Cell Longev 2019: 6175804, 2019.

31. Honda T, Hirakawa $\mathrm{Y}$ and Nangaku M: The role of oxidative stress and hypoxia in renal disease. Kidney Res Clin Pract 38: 414-426, 2019.

32. Kura B, Szeiffova Bacova B, Kalocayova B, Sykora M and Slezak J: Oxidative Stress-Responsive MicroRNAs in Heart Injury. Int J Mol Sci 21: 358, 2020.

33. Chen T, Li M, Fan X, Cheng J and Wang L: Sodium Tanshinone IIA Sulfonate Prevents Angiotensin II-Induced Differentiation of Human Atrial Fibroblasts into Myofibroblasts. Oxid Med Cell Longev 2018: 6712585, 2018.
34. Wang P, Zhou S, Xu L, Lu Y, Yuan X, Zhang H, Li R, Fang J and Liu P: Hydrogen peroxide-mediated oxidative stress and collagen synthesis in cardiac fibroblasts: Blockade by tanshinone IIA. J Ethnopharmacol 145: 152-161, 2013.

35. Wang P, Wu X, Bao Y, Fang J, Zhou S, Gao J, Pi R, Mou YG and Liu P: Tanshinone IIA prevents cardiac remodeling through attenuating NAD $(\mathrm{P}) \mathrm{H}$ oxidase-derived reactive oxygen species production in hypertensive rats. Pharmazie 66: $517-524,2011$

36. Huang L, Zhu J, Zheng M, Zou R, Zhou Y and Zhu M: Tanshinone IIA protects against subclinical lipopolysaccharide induced cardiac fibrosis in mice through inhibition of NADPH oxidase. Int Immunopharmacol 60: 59-63, 2018.

37. Wu DM, Wang YJ, Han XR, Wen X, Li L, Xu L, Lu J and Zheng YL: Tanshinone IIA prevents left ventricular remodelling via the TLR4/MyD88/NF- $\mathrm{BB}$ signalling pathway in rats with myocardial infarction. J Cell Mol Med 22: 3058-3072, 2018.

(i) (9) This work is licensed under a Creative Commons cc) Attribution-NonCommercial-NoDerivatives 4.0 International (CC BY-NC-ND 4.0) License. 